R. R. Diehl' ${ }^{1}$, R. Tavakolian ${ }^{2}$, E. Völzke ${ }^{3}$, T. Lempert ${ }^{3}$

${ }^{1}$ Klinik für Neurologie, Alfried Krupp Krankenhaus Rüttenscheid, Essen, Deutschland

${ }^{2}$ Berlin, Deutschland

${ }^{3}$ Neurologische Abteilung, Schlosspark-Klinik, Berlin, Deutschland

\title{
Unabhängiges Informationsportal?
}

\section{Leserbrief zu}

Biogen-Anzeige (2019) Unabhängiges Informationsportal - Mit Neurodiem auf dem neuesten Stand. DGNeurologie Ausgabe 5/2019

\section{Erwiderungen}

Schmitt A (2020) Wissenstransfer ist in der Medizin ein zentraler Aspekt. https://doi.org/10.1007/s42451-020-00181-x Meyer zu Tittingdorf J (2020) Streben nach Transparenz. https://doi.org/ 10.1007/s42451-020-00180-y

Zunächst einmal: Gratulation zu DGNeurologie, dem besten deutschsprachigen Neurologiejournal! Anlass unseres Leserbriefs ist jedoch ein kritischer Punkt: Arbeitet man sich durch das Septemberheft der DGNeurologie, so gelangt man im ersten Drittel zu einem zweiseitigen Beitrag über die neue Informationsplattform Neurodiem. Dieser Bericht kommt in der Form wie eine Mitteilung der Redaktion daher. Schon im Titel wird die Unabhängigkeit des Portals herausgestellt. Sechs abgebildete Neurologen bürgen mit Bild und einem positiven Statement für die Qualität der neuen Website. Erst auf den 2. oder 3. Blick entdeckt man kleingedruckt im Seitenstreifen, dass es sich gar nicht um eine Empfehlung von DGNeurologie handelt, sondern um eine Anzeige des Pharmaunternehmens Biogen. „Ein Service von Biogen" heißt es auch auf www.neurodiem.de.

Die inhaltliche Qualität von Neurodiem können wir noch nicht beurteilen. Auf den ersten Blick präsentiert die Website relevante Studienergebnisse in ansprechender Form. Dennoch bleibt ein solches Portal aus Sicht unserer Initiative NeurologyFirst problematisch. Eine Anzeige eines Pharmaunternehmens sollte auf den ersten Blick als solche erkennbar sein und sich nicht als redaktioneller Beitrag tarnen. Auch wenn die Autoren laut den redaktionellen Richtlinien von Neurodiem frei bestimmen können, welche Inhalte sie einbringen, ist das Stichwort unabhängig irreführend. Die Informationsplattform Neurodiem ist durch die alleinige Finanzierung auf elementare Weise von Biogen abhängig. Echte Unabhängigkeit schließt die Freiheit ein, die Firma Biogen zu kritisieren, beispielsweise für ihre Preispolitik, die vielen Patienten, v. a. in ärmeren Ländern, den Zugang zur Behandlung versperrt, oder auch für das offensive Marketing von Daclizumab in Deutschland. Zur Erinnerung: Das MS-Medikament musste 2018 wegen mehrerer Todesfälle vom Markt genommen werden. Schon zum Zulassungszeit-

DGNeurologie 2020 · 3 (3): 269

https://doi.org/10.1007/s42451-020-00179-5

(c) Springer Medizin Verlag GmbH, ein Teil von Springer Nature 2020 punkt war das Sicherheitsprofil umstritten. Können wir diese Unabhängigkeit von Neurodiem erwarten? Weitere Zweifel regen sich angesichts der langjährigen finanziellen Beziehungen einiger der 6 neurologischen Gewährsmänner zu Biogen. In den Händen der DGN wäre ein ansprechendes neurologisches Webmagazin sicher besser aufgehoben als bei einer Firma mit kommerziellen Interessen! Wünschenswert wäre zudem ein redaktioneller Einfluss der DGNeurologie-Schriftleitung auf die kommerziellen Beilagen.

\section{Korrespondenzadresse}

Prof. Dr. R. R. Diehl

Klinik für Neurologie

Alfried Krupp Krankenhaus Rüttenscheid

Alfried-Krupp-Str. 21, 45131 Essen, Deutschland

rolf.diehl@krupp-krankenhaus.de

Interessenkonflikt. R. R. Diehl, R. Tavakolian, E. Völzke und T. Lempert geben an, dass kein Interessenkonflikt besteht. 